\title{
Impacto del tiempo de evolución del trauma cerrado de abdomen en el rendimiento diagnóstico del FAST (Focussed Assessment with Sonography for Trauma)
}

\author{
José M. Llamas-Montes ${ }^{1 *}$, Valery Melnikov² y Emilio Prieto-Díaz-Chávez ${ }^{2}$ \\ ${ }^{1}$ Servicio de Urgencias de Adultos, Nuevo Hospital Civil de Guadalajara Dr. Juan I. Menchaca, Guadalajara, Jalisco; ${ }^{2}$ Facultad de Medicina, \\ Universidad de Colima, Colima, Colima México
}

\section{Resumen}

Introducción: En el trauma cerrado de abdomen el riesgo de que el paciente muera aumenta considerablemente si la atención se retrasa cinco horas o más. Por eso es crucial determinar de forma rápida la necesidad de realizar una laparotomía urgente. Métodos: Estudio observacional, prospectivo. Muestreo consecutivo. Se calcularon la precisión, sensibilidad, especificidad, valor predictivo positivo y valor predictivo negativo, intervalo de confianza del 95\% y grado de significación (p) < 0.05. Con el programa IBM SPSS Statistics v.26 se desarrolló análisis de la varianza simple y pruebas post hoc para determinar la significancia estadística entre las diferencias según el tiempo de evolución de los pacientes. Resultados: Cuando los pacientes tuvieron una evolución menor de cinco horas desde que ocurrió el trauma la precisión, sensibilidad, especificidad, valor predictivo positivo y negativo fueron del 100\%, sin embargo, pasado este tiempo disminuye el rendimiento diagnóstico del FAST (focussed assessment with sonography for trauma). Conclusión: Clásicamente para la toma de decisiones en los pacientes con trauma cerrado de abdomen se tienen en consideración el estado del paciente y el resultado del FAST, sin embargo, es importante tener en cuenta el tiempo de evolución, pues este impacta en el rendimiento diagnóstico del FAST.

Palabras clave: Ultrasonido FAST. Trauma cerrado de abdomen. Evolución del trauma abdominal. Rendimiento diagnóstico del FAST.

Impact of the evolution time of blunt abdominal trauma on the diagnostic performance of FAST (Focussed Assessment with Sonography for Trauma)

\begin{abstract}
Introduction: in blunt abdominal trauma, the risk of death increases considerably if care is delayed for 5 hours or more. This is why it's crucial to quickly determine the need for an urgent laparotomy. Methods: prospective, observational study. Consecutive sampling. Accuracy, sensitivity, specificity, positive predictive value and negative predictive value, confidence interval with $p$ value of 0.05 were calculated. With IBM SPSS Statistics v.26 program, simple ANOVA and post hoc test were developed to determine the significance between the differences according to evolution time of patients. Results: when patients had an evolution less than 5 hours since the trauma occurred, the accuracy, sensitivity, specificity, positive and negative predictive value were $100 \%$, however, after this time the diagnostic performance of FAST decreases. Conclusion: classically,
\end{abstract}

\section{Correspondencia:}

*José M.A. Llamas-Montes

E-mail: llamas_montes@ hotmail.com

Disponible en internet: $25-11-2021$

Fecha de recepción: 18-06-2021

Fecha de aceptación: 22-07-2021 DOI: 10.24875/REIE.21000063
Rev Educ Investig Emer. 2021;3(4):189-197 www.medicinadeemergencias.com

2604-6520 (c) 2021 Sociedad Mexicana de Medicina de Emergencias, AC. Publicado por Permanyer México SA de CV. Este es un artículo open access bajo la licencia CC BY-NC-ND (http://creativecommons.org/licenses/by-nc-nd/4.0/). 
for decision-making in patients with blunt abdominal trauma, the patient's condition and the result of the FAST are taken into consideration, however, it's important to take into account the evolution time, as it impacts the diagnostic performance of the FAST.

Key words: FAST ultrasound. Blunt abdominal trauma. Evolution of abdominal trauma. Diagnostic performance of FAST.

\section{Introducción}

El trauma cerrado de abdomen puede pasar desapercibido debido a que la exploración física tiene una sensibilidad variable, entre el 47 y el $87 \%$, dependiendo de muchos factores. Muchos de los signos y síntomas no siempre se manifiestan (Tabla 1), especialmente cuando el paciente cursa con un deterioro del estado de alerta, déficit neurológico, otras lesiones asociadas o se encuentra bajo los efectos de drogas, sustancias o medicamentos ${ }^{1,2}$.

El traumatismo abdominal cerrado representa el $75 \%$ de todos los traumas cerrados. Sus principales causas son accidentes vehiculares, atropellamiento, caídas, agresiones, lesiones durante deportes 0 actividades recreativas y accidentes con objetos inusuales ${ }^{1,3}$.

En el trauma cerrado de abdomen la prevalencia de lesiones intraabdominales es entre el 12 y el 15\%, con una tasa de mortalidad del 8 al $25 \%$, que se encuentra en estrecha relación con el retraso en el diagnóstico, reportándose actualmente que el riesgo de que el paciente muera aumenta considerablemente si la atención se retrasa cinco horas o más, pues se estima que aumenta aproximadamente un $1 \%$ por cada tres minutos que se retrase la cirugía en estos pacientes, por lo que es potencialmente prevenible si el diagnóstico se establece de forma rápida ${ }^{4-7}$. Por eso es crucial determinar de forma rápida la necesidad de realizar una laparotomía urgente, especialmente en los pacientes con inestabilidad hemodinámica ${ }^{3,5}$.

El ultrasonido se ha vuelto una herramienta diagnóstica ampliamente usada en el contexto de atención de pacientes con trauma, sin embargo, no es práctico que lo realice un radiólogo por el tiempo que puede tomar llevar al paciente a la sala de radiología o que el radiólogo acuda a urgencias, lo que puede generar retraso en el manejo del paciente ${ }^{8,9}$. Es por lo anterior que el ultrasonido de abdomen actualmente es el primer método de imagen para el cribado de pacientes con trama cerrado de abdomen y se ha convertido en una extensión de la exploración física, formando parte de la evaluación inicial del Advanced Trauma Life Support (ATLS®) 3,10,11.

Un protocolo de ultrasonido usado para la detección de líquido libre intraperitoneal en el contexto de trauma cerrado de abdomen es el FAST (focussed assessment
Tabla 1. Porcentaje de presentación de signos clínicos encontrados a la exploración física del paciente con trauma cerrado de abdomen ${ }^{1}$

\begin{tabular}{|l|c|}
\hline Signos clínicos & Porcentaje \\
\hline Abrasión/contusión & $58 \%$ \\
\hline Hipersensibilidad & $40 \%$ \\
\hline Ausencia de ruidos abdominales & $35 \%$ \\
\hline Defensa muscular & $34 \%$ \\
\hline Rigidez abdominal & $23 \%$ \\
\hline Distensión abdominal & $17 \%$ \\
\hline Matidez a la percusión & $17 \%$ \\
\hline Sangre en meato urinario & $2 \%$ \\
\hline $\begin{array}{l}\text { Edema o hematoma en periné, vagina, recto o } \\
\text { glúteos }\end{array}$ & $1 \%$ \\
\hline
\end{tabular}

Adaptada de Pancholi, et al., 2017 .

with sonography for trauma), que tiene como objetivo primordial el de responder la siguiente pregunta: ¿existe líquido libre en la cavidad peritoneal y/o pericárdica o no? ?2-14 $^{12}$

EI FAST es una prueba que ofrece bastantes ventajas: es rápido, portátil, útil, no invasivo, reproducible, seguro, puede realizarse durante las maniobras de reanimación y manejo iniciales, se realiza en la cama del paciente, entre otras $2,12,13,15$. Sin embargo, también tiene desventajas: es operador dependiente, se necesita entrenamiento por parte del operador, tiene limitación para valorar retroperitoneo e incluso pelvis, alto costo del equipo, entre otras $2,8,13,15$.

Realizar un FAST a un paciente con trauma cerrado de abdomen no toma más de cuatro minutos e incluso puede ser realizado en menos de dos minutos, con la capacidad de detectar a partir de $200 \mathrm{ml}$ de líquido libre intraperitoneal dependiendo de la experiencia del operador ${ }^{1,2,16}$.

Se ha reportado que el FAST realizado por urgenciólogos tiene una sensibilidad del $50-78.5 \%$, especificidad de $97.3-99 \%$, valor predictivo positivo y negativo del 87 y $84 \%$ respectivamente, con una precisión del $85 \%$, con una mayor sensibilidad en pacientes de 
menor gravedad y una mayor especificidad a mayor gravedad $^{12,14}$.

La tasa de falso negativo es del $1-12.9 \%$, que puede ser debido a varios factores, entre los cuales, el tiempo de realización del estudio juega un papel importante. De tal manera tanto una realización del estudio demasiado temprana como su retraso excesivo son perjudiciales. En el primer caso se reporta un alto porcentaje de pacientes que requieren de tiempo para la acumulación del líquido intraperitoneal en cantidades suficientes para ser detectados. En caso de retraso los cambios propios por el trauma trauma pueden enmascarar el líquido intraperitoneal. Otros factores que intervienen son adherencias, obesidad, exceso de luz ambiental, entre otras causas $^{6,17-20}$. De igual modo, errores en la interpretación de los hallazgos encontrados en el FAST y su correlación con el contexto clínico del paciente pueden llevar a falsos positivos, con una tasa que se encuentra alrededor del $22 \%$, entre los que se encuentran: grasa perirrenal, ascitis, vísceras con líquido intraluminal como el estómago o el intestino y más ${ }^{18,20}$.

En el FAST pueden pasar inadvertidas lesiones que requieren tratamiento quirúrgico inmediato, por lo que no sustituye a la tomografía de abdomen, que tiene mayor sensibilidad y especificidad para detectar lesiones intraabdominales y líquido libre, sin embargo, realizar una tomografía puede llevar como mínimo 3-4 minutos dependiendo de las circunstancias de la unidad hospitalaria y la organización del personal, por lo que entre más cercano este el tomógrafo a la sala de urgencias más posibilidades de supervivencia tiene el paciente, disminuyendo esta cuando la distancia entre el servicio de urgencias y el equipo tomográfico es mayor a 50 metros $5,7,8,12,14$. Además, por lo general el traslado de pacientes a tomografía se contraindica de manera relativa hasta después de realizar las maniobras de reanimación, especialmente cuando no se puede determinar el origen de la hemorragia con los estudios de imagen iniciales, esto para minimizar los riesgos de descompensación durante el traslado ${ }^{15}$.

EI FAST debe ser usado solo para determinar que pacientes con trauma cerrado de abdomen deben ser tratados quirúrgicamente de forma inmediata o pueden ser abordados con otros estudios de imagen y plantear un tratamiento conservador, según los resultados del FAST y el estado hemodinámico del paciente: si el resultado del FAST es positivo, especialmente si se encuentra una cantidad considerable de líquido libre, está justificado que el paciente se someta a laparotomía exploradora, más aún cuando se encuentra con inestabilidad hemodinámica, reduciendo así el tiempo entre la sala de urgencias y el quirófano. Por el contrario, si el resultado es negativo y el paciente está estable hemodinámicamente o mejora el estado hemodinámico, debe realizarse una tomografía de abdomen para determinar si existe o no lesión intraabdominal $5,9,14,15,18,21$. Cabe destacar que el FAST tiene una buena sensibilidad y especificidad para la detección de hemoperitoneo, por lo que es una herramienta válida en el abordaje inicial de todo paciente traumatizado, pues un examen positivo con frecuencia requiere cirugía, reportándose correlación entre el FAST y la laparotomía en el $64 \%$ de los casos. Por el contrario, cuando el resultado es negativo con mucha seguridad se puede excluir una lesión que requiera manejo quirúrgico inmediato, con lo que se ha reducido la tasa de laparotomías. Sin embargo, no se descarta la existencia de una lesión intraabdominal que debe investigarse con estudios de mayor rendimiento diagnóstico, por lo que su principal ventaja clínica es tal vez la de ahorrar tiempo en el abordaje de los pacientes con trauma cerrado de abdomen $7,10,16,18,21,22$.

Se han realizado pocos estudios en los que se evalúe el rendimiento del FAST en el paciente con trauma cerrado de abdomen según el tiempo de evolución que haya transcurrido desde la lesión hasta la realización del mismo, corroborando sus hallazgos por medio de tomografía o cirugía abdominal.

El objetivo de este estudio es determinar el impacto que tiene, el tiempo transcurrido entre el traumatismo y la atención de los pacientes, en el rendimiento diagnóstico del FAST.

\section{Material y métodos}

Estudio observacional, prospectivo. Muestreo consecutivo por conveniencia: se incluyeron todos los pacientes con trauma cerrado de abdomen que ingresaron al servicio de urgencias adultos del Antiguo Hospital Civil de Guadalajara Fray Antonio Alcalde del 1 de abril al 30 de septiembre de 2019.

Criterios de inclusión: a) pacientes de 15 años de edad en adelante, que hubiesen sufrido trauma abdominal en los últimos siete días, b) que al paciente se le realizara FAST durante su abordaje en el servicio de urgencias. Criterios de no inclusión: a) paciente con trauma penetrante de abdomen, b) paciente conocido con ascitis o enfermedad renal crónica, c) pacientes embarazadas, d) pacientes que hayan sido sometidos a cirugía abdominal en el último mes. Criterios de exclusión: a) pacientes a quienes no se les haya realizado tomografía computarizada de abdomen y/o laparotomía exploratoria dentro del abordaje diagnóstico-terapéutico. 
Se utilizo un equipo de ultrasonido modelo Sonoline Adara $^{\circledR}$ de la marca Siemens (Alemania), con transductor convexo de $3.5 \mathrm{MHz}$.

Al ingreso de los pacientes se les realizó la evaluación primaria del $\operatorname{ATLS}^{\circledR}$ y posteriormente, ya sea que se lograra o no la estabilización hemodinámica, se realizó el protocolo FAST, registrando los resultados en una base de datos diseñada para el estudio, por lo que la atención de estos pacientes no fue retrasada para el registro de datos.

Únicamente se incluyeron las ventanas de ultrasonido FAST de abdomen: hepatorrenal, esplenorrenal y hueco pélvico. A todos los pacientes se les realizó tomografía de abdomen independientemente del resultado del ultrasonido FAST, salvo aquellos a los que se les llevó a quirófano de forma inmediata. Todos los pacientes fueron valorados por el servicio de cirugía (cirugía medicina legal, para efectos del centro hospitalario).

Se calcularon la precisión, sensibilidad, especificidad, valor predictivo positivo y valor predictivo negativo, según el tiempo de evolución transcurrido desde que ocurrió el traumatismo hasta que se realizó el FAST, con un intervalo de confianza con valor de $p$ de 0.05 . Con el programa IBM SPSS Statistics v. 26 se desarrolló análisis de la varianza (ANOVA) simple y pruebas post hoc para determinar la significancia estadística entre las diferencias según el tiempo de evolución de los pacientes.

Por tratarse de un estudio descriptivo no se realizaron intervenciones ni modificaciones al proceso de abordaje diagnostico-terapéutico de los pacientes. Cumple con los principios establecidos por la World Medical Association en la Declaración de Helsinki actualizada en 2013, que también se encuentran contemplados en la Ley General de Salud título quinto, articulo 100.

\section{Resultados}

Durante el periodo de captura se registraron 56 pacientes con trauma cerrado de abdomen que cumplían con los criterios de inclusión de los cuales 10 fueron excluidos del estudio por no habérseles realizado tomografía de abdomen ni laparotomía durante su abordaje, incluyendo finalmente 46 pacientes. De los pacientes incluidos, 40 fueron masculinos, con un promedio de edad de 34 años (Tabla 2). El tiempo de evolución desde que los pacientes sufrieron el trauma hasta que se les atendió en el servicio de urgencias fue en promedio 16 horas, presentándose únicamente ocho pacientes
Tabla 2. Características generales de los pacientes incluidos en el estudio

\begin{tabular}{|c|c|}
\hline Características & $n(\%)$ \\
\hline $\begin{array}{l}\text { Total de pacientes } \\
\text { Hombres } \\
\text { Mujeres }\end{array}$ & $\begin{array}{c}46(100 \%) \\
40(87 \%) \\
6(13 \%)\end{array}$ \\
\hline $\begin{array}{l}\text { Edad promedio en años } \\
\begin{array}{l}\text { 15-19 años } \\
\text { 20-29 años } \\
30-39 \text { años } \\
40-49 \text { años } \\
50-59 \text { años } \\
60-69 \text { años } \\
70-79 \text { años }\end{array}\end{array}$ & $\begin{array}{c}34(15-79) \\
13(28.2 \%) \\
9(19.5 \%) \\
8(17.4 \%) \\
6(13 \%) \\
9(19.5 \%) \\
0 \\
1(2.2 \%)\end{array}$ \\
\hline $\begin{array}{l}\text { Tiempo de evolución promedio en horas } \\
<5 \text { horas } \\
5-12 \text { horas } \\
13-24 \text { horas } \\
>24 \text { horas }\end{array}$ & $\begin{array}{c}16(2-144) \\
8(17.4 \%) \\
22(47.8 \%) \\
12(26.1 \%) \\
4(8.7 \%)\end{array}$ \\
\hline $\begin{array}{l}\text { Mecanismos de lesión } \\
\text { Accidente en motocicleta } \\
\text { Atropellamiento } \\
\text { Agresión con contundente } \\
\text { Accidente en automóvil } \\
\text { Caída de altura } \\
\text { Aplastamiento por tronco } \\
\text { Accidente en camión } \\
\text { Pisado por caballo } \\
\text { Pisado por toro }\end{array}$ & $\begin{array}{c}17(36.9 \%) \\
7(15.2 \%) \\
7(15.2 \%) \\
6(13 \%) \\
5(10.8 \%) \\
1(2.1 \%) \\
1(2.1 \%) \\
1(2.1 \%) \\
1(2.1 \%)\end{array}$ \\
\hline
\end{tabular}

con menos de cinco horas de evolución y una mayor cantidad de pacientes entre las 5 y las 12 horas de evolución, disminuyendo drásticamente después de las 24 horas de evolución (Tabla 2). La causa más común de trauma cerrado de abdomen que se encontró en el presente estudio fue accidente en motocicleta, seguida de atropellamiento y agresión con objeto contundente (Tabla 2)

De los 46 pacientes incluidos, el FAST resultó positivo en 20 (43.4\%) (Fig. 1 A y B) y negativo en 26 (56.6\%) (Fig. 1C); a 41 (89.1\%) pacientes se les realizó tomografía de abdomen, de las cuales 20 (48.7\%) fueron positivas (Fig. 1D) y $21(51.2 \%)$ negativas para líquido libre en cavidad abdominal; 15 (32.6\%) pacientes fueron sometidos a laparotomía exploratoria, resultando $13(86.6 \%)$ positivas y $2(13.3 \%)$ negativas para líquido libre.

De los ultrasonidos con resultado positivo la ventana en que se evidenció el líquido libre con mayor frecuencia fue la hepatorrenal, con el $60 \%$ de los casos, seguida de la de hueco pélvico con un $45 \%$ y la esplenorrenal con un $35 \%$. 


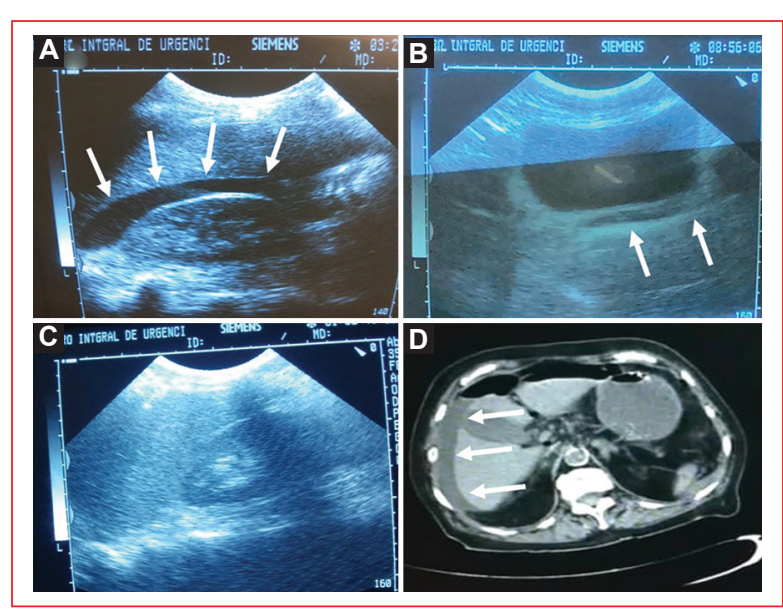

Figura 1. A: FAST positivo (ventana hepatorrenal). B: FAST positivo (ventana de hueco pélvico). C: FAST negativo (ventana hepatorrenal). D: tomografía simple de abdomen, las flechas blancas indica imagen hipoecoica compatible con líquido libre.

Se calculó una precisión global del $86.9 \%$, sensibilidad del $79.1 \%$, especificidad del $95.4 \%$, valor predictivo positivo del $95 \%$ y valor predictivo negativo del $80.7 \%$ (Tabla 3).

Cuando los pacientes tuvieron una evolución menor de cinco horas desde que ocurrió el trauma, la precisión, sensibilidad, especificidad, valor predictivo positivo y negativo fueron del $100 \%$, sin embargo, pasado este tiempo disminuye el rendimiento diagnóstico del FAST (Tabla 3). Se encontraron cinco falsos negativos y solo un falso positivo (Fig. 2), todos en el grupo de cinco horas o más de evolución, con lo cual se calcularon tasas del 20.1 y el $4.6 \%$, respectivamente (Tabla 3).

Se calculó la diferencia entre la precisión, sensibilidad, especificidad y valores predictivos positivo y negativo según el tiempo de evolución de los pacientes. La diferencia fue estadísticamente significativa únicamente en los resultados del valor predictivo negativo obtenidos entre las 5-12 horas y 13-24 horas al compararlos con los obtenidos después de las 24 horas. En pacientes con menos de cinco horas no hubo diferencias estadísticamente significativas al compararlas con ningún grupo de pacientes. En el resto de comparaciones no se encontró significancia estadística (Tabla 4).

\section{Discusión}

Según datos reportados por el Instituto Nacional de Estadística y Geografía (INEGI), los accidentes vehiculares son de las principales causas de mortalidad en
México y los sufren principalmente hombres jóvenes, datos que concuerdan con los resultados de nuestro estudio, puesto que se encontró que la mayoría de pacientes atendidos por trauma cerrado de abdomen fueron hombres menores de 40 años por accidente en motocicleta ${ }^{23}$.

En cuanto al tiempo de evolución transcurrido desde sufrir la lesión hasta presentarse al servicio de urgencias y que se le realizara el FAST, se encontró que la mayor cantidad de pacientes se presentaron entre las 5 y las 12 horas posterior al accidente, lo que crea una necesidad imperativa de estudios enfocados en detectar las causas de tal retraso en la etapa prehospitalaria de atención.

Tajjodini, et al. realizaron en 2013 un estudio prospectivo en el que se comparó la precisión de los FAST realizados por urgenciólogos y por radiólogos, encontrando que la ventana hepatorrenal es la que con mayor frecuencia es más fácil de explorar y la que muestra una mayor tasa de resultados que las demás ventanas, posiblemente esto se debe a que el riñón derecho se encuentra en una posición más inferior que el izquierdo y a que en el lado izquierdo se interpone el colon en la zona de insonación; también reportaron que la precisión es similar en ambos grupos de especialistas ${ }^{22}$, lo que claramente concuerda con los resultados de este estudio. Sin embargo, la ventana hepatorrenal a pesar de ser la que más permitió visualizar líquido libre también fue la única que mostró un caso de falso positivo, lo cual tiene múltiples explicaciones, como que al ser las otras ventanas más complejas de valorar se cuidan más los detalles y se analizan con mayor detenimiento, al contrario de la ventana hepatorrenal.

Por otro lado, en la bibliografía se encuentran estudios similares al nuestro, desde series pequeñas, como la nuestra, hasta grandes muestras de pacientes. Kumar, et al. publicaron en 2012 un estudio prospectivo con una muestra de 50 pacientes de trauma cerrado de abdomen, reportando precisión del $80 \%$, sensibilidad del $80.4 \%$, especificidad del $75 \%$, valor predictivo positivo del $97.3 \%$ y valor predictivo negativo del $27.2 \%{ }^{10}$. Una de las series más grandes es la publicada en 2001 por Richards, et al., en la que de forma prospectiva integran a 3,264 pacientes, obteniendo como resultados precisión del $94 \%$, sensibilidad del $60 \%$, especificidad del $98 \%$, valor predictivo positivo del $82 \%$ y valor predictivo negativo del $95 \%^{24}$.

Concretamente analizando el objetivo principal de este estudio que es el de determinar si el tiempo de 
Tabla 3. Resultados globales y por tiempo de evolución de los pacientes

\begin{tabular}{|c|c|c|c|c|c|}
\hline Grupo & Global & $<5$ horas & $5-12$ horas & 13-24 horas & $>24$ horas \\
\hline N. ${ }^{0}$ total de FAST & $46(100 \%)$ & $8(17.4 \%)$ & $22(47.8 \%)$ & $12(26.1 \%)$ & $4(8.7 \%)$ \\
\hline N. ${ }^{\circ}$ FAST positivo & $20(43.5 \%)$ & $6(13 \%)$ & $6(13 \%)$ & $5(10.9 \%)$ & $3(6.5 \%)$ \\
\hline N. ${ }^{0}$ FAST negativo & $26(56.5 \%)$ & $2(4.4 \%)$ & $16(34.8 \%)$ & $7(15.2 \%)$ & $1(2.2 \%)$ \\
\hline N. ${ }^{\circ}$ gold standard positivo & $24(52.2 \%)$ & $6(13 \%)$ & $9(19.5 \%)$ & $5(10.9 \%)$ & $4(8.7 \%)$ \\
\hline N. ${ }^{\circ}$ gold standard negativo & $22(47.8 \%)$ & $2(4.4 \%)$ & $13(28.3 \%)$ & $7(15.2 \%)$ & 0 \\
\hline N..$^{\circ}$ FAST verdadero positivo & $19(41.3 \%)$ & $6(13 \%)$ & $6(13 \%)$ & $4(8.7 \%)$ & $3(6.5 \%)$ \\
\hline N. ${ }^{0}$ FAST falso positivo & $1(2.2 \%)$ & 0 & 0 & $1(2.2 \%)$ & 0 \\
\hline N. ${ }^{0}$ FAST falso negativo & $5(10.9 \%)$ & 0 & $3(6.5 \%)$ & $1(2.2 \%)$ & $1(2.2 \%)$ \\
\hline N. ${ }^{\circ}$ FAST verdadero negativo & $21(45.6 \%)$ & $2(4.4 \%)$ & $13(28.3 \%)$ & $6(13 \%)$ & 0 \\
\hline Precisión \% & 86.9 (IC: 77.2-96.3) & 100 & 86.3 (IC: $72-100)$ & 83.3 (IC: $62.2-100$ ) & 75 (IC: $32.6-100)$ \\
\hline Sensibilidad \% & 79.1 (IC: 62.9-95.3) & 100 & 66.6 (IC: $35.8-97.4)$ & 80 (IC: $45-100)$ & 75 (IC: $32.6-100)$ \\
\hline Especificidad \% & 95.4 (IC: $86.7-100)$ & 100 & 100 & 85.7 (IC: $59.8-100$ ) & * \\
\hline Valor predictivo positivo \% & 95 (IC: $85.5-100)$ & 100 & 100 & 80 (IC: $45-100)$ & 100 \\
\hline Valor predictivo negativo \% & 80.7 (IC: 65.6-95.8) & 100 & 81.2 (IC: $62.1-100)$ & 85.7 (IC: $59.8-100$ ) & 0 \\
\hline Tasa falsos negativos \% & 20.9 & 0 & 33.4 & 20 & 25 \\
\hline Tasa falsos positivos $\%$ & 4.6 & 0 & 0 & 14.3 & * \\
\hline
\end{tabular}

*Resultado no calculable.

FAST: focussed assessment with sonography for trauma; IC: intervalo de confianza.

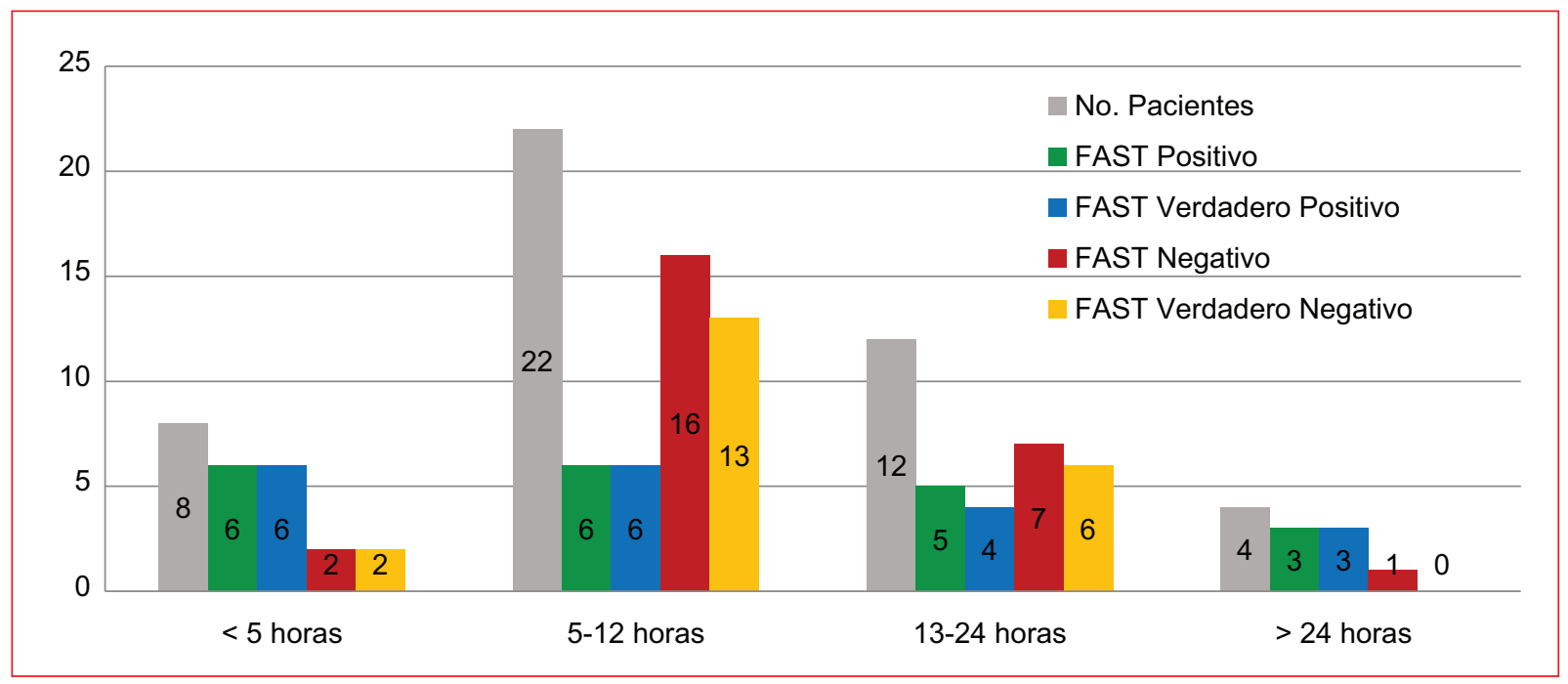

Figura 2. Resultados de los cálculos estadísticos según el tiempo de evolución de los pacientes.

evolución impacta en los resultados del FAST y por ende en el rendimiento diagnóstico de este, los estudios publicados concuerdan en que después de cierto tiempo de evolución posterior al traumatismo el FAST pierde rendimiento diagnóstico, sin embargo, son muy pocos los que abordan en profundidad cuál es la magnitud de dicho impacto. Al respecto, Richards y McGahan exponen que los pacientes con trauma cerrado abdominal que se presentan de manera retrasada pueden tener hemoperitoneo que contenga $o$ que se ha 
Tabla 4. Diferencia entre los resultados según el tiempo de evolución del paciente

\begin{tabular}{|c|c|c|c|c|c|c|c|c|c|c|}
\hline \multirow[t]{2}{*}{ Comparación } & \multicolumn{2}{|c|}{ Precisión } & \multicolumn{2}{|c|}{ Sensibilidad } & \multicolumn{2}{|c|}{ Especificidad } & \multicolumn{2}{|c|}{$\begin{array}{c}\text { Valor predictivo } \\
\text { positivo }\end{array}$} & \multicolumn{2}{|c|}{$\begin{array}{c}\text { Valor predictivo } \\
\text { negativo }\end{array}$} \\
\hline & Diferencia & $\mathbf{p}$ & Diferencia & $\mathbf{p}$ & Diferencia & $\mathbf{p}$ & Diferencia & $\mathbf{p}$ & Diferencia & $\mathbf{p}$ \\
\hline $\begin{array}{l}<5 \text { horas } \\
5-12 \text { horas } \\
13-24 \text { horas } \\
>24 \text { horas }\end{array}$ & $\begin{array}{c}13.7 \\
16.7 \\
25\end{array}$ & $\begin{array}{l}0.77 \\
0.71 \\
0.64\end{array}$ & $\begin{array}{c}33.4 \\
20 \\
25\end{array}$ & $\begin{array}{l}0.45 \\
0.86 \\
0.79\end{array}$ & $\begin{array}{c}0 \\
14.3 \\
*\end{array}$ & $\begin{array}{c}1.0 \\
0.68 \\
*\end{array}$ & $\begin{array}{c}0 \\
20 \\
0\end{array}$ & $\begin{array}{c}1 \\
0.47 \\
1\end{array}$ & $\begin{array}{l}18.8 \\
14.3 \\
100\end{array}$ & $\begin{array}{l}0.91 \\
0.96 \\
0.06\end{array}$ \\
\hline $\begin{array}{l}5-12 \text { horas } \\
<5 \text { horas } \\
13-24 \text { horas } \\
>24 \text { horas }\end{array}$ & $\begin{array}{c}-13.7 \\
3 \\
11.3\end{array}$ & $\begin{array}{l}0.77 \\
0.99 \\
0.93\end{array}$ & $\begin{array}{c}-33.4 \\
-13.4 \\
-8.4\end{array}$ & $\begin{array}{l}0.45 \\
0.94 \\
0.98\end{array}$ & $\begin{array}{c}0 \\
14.3 \\
*\end{array}$ & $\begin{array}{c}1 \\
0.34 \\
*\end{array}$ & $\begin{array}{c}0 \\
20 \\
0\end{array}$ & $\begin{array}{c}1 \\
0.47 \\
1\end{array}$ & $\begin{array}{c}-18.8 \\
-4.5 \\
81.2\end{array}$ & $\begin{array}{l}0.91 \\
0.99 \\
0.04\end{array}$ \\
\hline $\begin{array}{l}13-24 \text { horas } \\
<5 \text { horas } \\
5-12 \text { horas } \\
>24 \text { horas }\end{array}$ & $\begin{array}{c}-16.7 \\
-3 \\
8.3\end{array}$ & $\begin{array}{l}0.71 \\
0.99 \\
0.97\end{array}$ & $\begin{array}{c}-20 \\
13.4 \\
5\end{array}$ & $\begin{array}{l}0.86 \\
0.94 \\
0.99\end{array}$ & $\begin{array}{c}-14.3 \\
-14.3 \\
*\end{array}$ & $\begin{array}{c}0.68 \\
0.34 \\
*\end{array}$ & $\begin{array}{l}-20 \\
-20 \\
-20\end{array}$ & $\begin{array}{l}0.47 \\
0.47 \\
0.62\end{array}$ & $\begin{array}{c}-14.3 \\
4.5 \\
85.7\end{array}$ & $\begin{array}{l}0.96 \\
0.99 \\
0.04\end{array}$ \\
\hline $\begin{aligned}> & 24 \text { horas } \\
& <5 \text { horas } \\
& 5-12 \text { horas } \\
& 13-24 \text { horas }\end{aligned}$ & $\begin{array}{c}-25 \\
-11.3 \\
-8.3\end{array}$ & $\begin{array}{l}0.64 \\
0.93 \\
0.97\end{array}$ & $\begin{array}{c}-25 \\
8.4 \\
-5\end{array}$ & $\begin{array}{l}0.79 \\
0.98 \\
0.99\end{array}$ & $\begin{array}{l}* \\
* \\
*\end{array}$ & $\begin{array}{l}* \\
* \\
*\end{array}$ & $\begin{array}{c}0 \\
0 \\
20\end{array}$ & $\begin{array}{c}1 \\
1 \\
0.62\end{array}$ & $\begin{array}{c}-100 \\
-81.2 \\
-85.47\end{array}$ & $\begin{array}{l}0.06 \\
0.04 \\
0.04\end{array}$ \\
\hline
\end{tabular}

*Resultado no calculable.

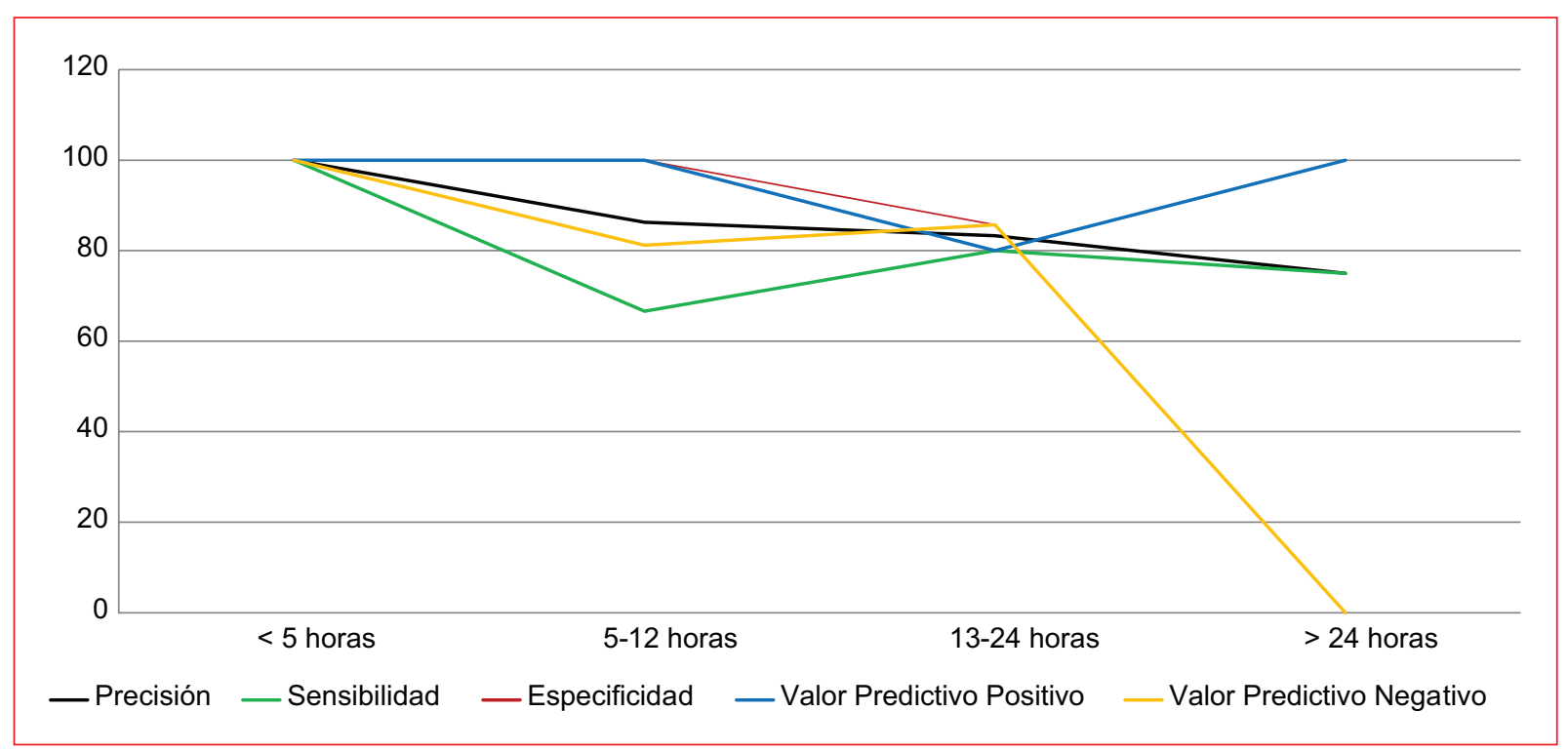

Figura 3. Resultados de los cálculos estadísticos según el tiempo de evolución de los pacientes.

convertido en coágulos, lo que puede generar que pase desapercibido el líquido libre ${ }^{25}$.

Nuestro estudio demostró que, al pasar el tiempo, disminuye la precisión del FAST, sin embargo, la sensibilidad, especificidad y valores predictivos sufren un comportamiento no lineal, puesto que forman una curva dicrótica: con un valor del $100 \%$ cuando la evolución de los pacientes es menor de 5 horas, disminuyendo entre las 5 y 12 horas, subiendo nuevamente entre las 13 y 24 horas y volviendo a disminuir después de las 24 horas. A fin de cuentas, a medida que pasa el tiempo el rendimiento diagnóstico del FAST es menor. Este comportamiento puede observarse en la figura 3, observando una pendiente descendente y posteriormente asciende nuevamente.

A pesar de que en aquellos pacientes que se atendieron antes de las cinco horas de evolución el FAST tuvo un rendimiento diagnóstico perfecto, no se encontró diferencia estadística significativa. Esto se explica al recalcar 
que aquellos pacientes con menos de cinco horas evolución son en su mayoría pacientes más graves y hemodinámicamente inestables, por lo que el resultado del ultrasonido FAST es más confiable en estos casos, como lo muestran los resultados publicados por Shek en $2012^{14}$.

La diferencia estadística fue significativa únicamente en el valor predictivo negativo, es decir, la probabilidad de que un FAST negativo sea un caso verdadero negativo, al comparar aquellos casos que se atendieron entre las 5 y 24 horas (5-12 horas y 13-24 horas) contra los que se atendieron después de las 24 horas, posiblemente debido a que después de las cinco horas el líquido que se ha acumulado es suficiente para ser detectado de manera confiable, pero después de las 24 horas este líquido puede sufrir cambios, como coagulación de sangre libre, que pueden llevar a un diagnóstico erróneo, además de que después de este tiempo lo más probable es que el paciente se encuentre estable hemodinámicamente, lo que permite realizar un FAST con más detenimiento y de manera cuidadosa y detallada.

Con lo anterior podemos decir que en caso de obtener un resultado negativo en el FAST debe tenerse en cuenta el tiempo que el paciente tiene de evolución, puesto que si se encuentra dentro de las primeras 24 horas puede brindar certeza en el diagnóstico, sin embargo, después de estas 24 horas un resultado negativo puede no ser confiable. Por el contrario, si el FAST es positivo, sin importar el tiempo de evolución, el paciente seguramente tendrá líquido libre abdominal, lo cual se refleja en la baja tasa de falsos positivos.

Cabe recalcar que todos los FAST deben ser corroborados por tomografía de abdomen, siempre que el paciente se encuentre estable hemodinámicamente y sea posible realizarla, con el fin de determinar si el paciente es candidato a un tratamiento conservador 0 debe ser intervenido quirúrgicamente.

Por último, cabe aclarar que los datos que no fueron posibles de calcular son aquellos relacionados con los pacientes atendidos después de 24 horas, los cuales son pocos, pero al tratarse de un estudio que analizó la relación del tiempo con el FAST es necesario incluirlos, teniendo en consideración que existen estudios publicados con muestras tan pequeñas como la nuestra.

\section{Conclusión}

Clásicamente para la toma de decisiones en los pacientes con trauma cerrado de abdomen se tienen en consideración el estado del paciente y el resultado del
FAST, sin embargo, es importante tener en cuenta el tiempo de evolución, pues este impacta en el rendimiento diagnóstico del FAST.

\section{Financiamiento}

Los autores declaran no haber recibido financiamiento para este estudio.

\section{Conflicto de intereses}

Los autores declaran no tener conflicto de intereses.

\section{Responsabilidades éticas}

Protección de personas y animales. Los autores declaran que para esta investigación no se han realizado experimentos en seres humanos ni en animales.

Confidencialidad de los datos. Los autores declaran que han seguido los protocolos de su centro de trabajo sobre la publicación de datos de pacientes.

Derecho a la privacidad y consentimiento informado. Los autores han obtenido el consentimiento informado de los pacientes y/o sujetos referidos en el artículo. Este documento obra en poder del autor de correspondencia.

\section{Bibliografía}

1. Pancholi K, Patel S, Parikh R, Saxena AK. Efficacy of Focused Assessment Sonography in Trauma (FAST) in patients with blunt abdominal trauma. International Research and Publication in Medical Sciences. 2017;2(5):1-8.

2. Piróg M, Putowski M, Padala O, Orzel A, Sorochynskyy I, Wdowiak A. The focused assessment with ultrasound for trauma (FAST) examination in emergency medicine. European Journal of Medical Technologies. 2017;2(15):45-50.

3. Malak Boutros S, Amin Nassef M, Fathy Abdel-Ghany A. Blunt abdominal trauma: the role of focused abdominal sonography in assessment of organ injury and reducing the need for CT. Alexandra Journal of Medicine. 2016;52:35-41.

4. Heidari K, Taghizadeh M, Mohmoudi S, Panahi H, Ghaffari Shad E, Asadallahi S. FAST for blunt abdominal trauma: Correlation between positive findings and admission acid-base measurement. Am J Emerg Med. 2017;35:823-9.

5. Grünherz L, Jensen KO, Neuhaus V, Mica L, Werner CML, Ciritsis B, et al. Early computed tomography or focused assessment with sonography in abdominal trauma: what are the leading opinions? Eur J Trauma Emerg Surg. 2018;44(1):3-8

6. Mohammadi A, Ghasemi-Rad M. Evaluation of gastrointestinal injury in blunt abdominal trauma "FAST is not reliable": the role of repeated ultrasonography. World J Emerg Surg. 2017;7(2):1-6.

7. Karki OB. The role of computed tomography in blunt abdominal trauma. J Nepal Med Assoc. 2015;53(200):227-30.

8. Akoglu H, Celik OF, Celik A, Ergelen R, Onur O, Denizbasi A. Diagnostic accuracy of the extended focused abdominal sonography for trauma (E-FAST) performed by emergency physicians compared to CT. Am J Emerg Med. 2018;36:1014-7.

9. Hsu SD, Chen CJ, Chan DC, Yu JC. Senior general surgery residents can be trained to perform focused assessment with sonography for trauma patients accurately. Surg Today. 2017;47(12):1443-9.

10. Kumar S, Kumar Bansal V, Kumar Muduly D, Sharma P, Misra MC, et al. Accuracy of focused assessment with sonography for trauma (FAST) in blunt trauma abdomen - A prospective study. Indian J Surg. 2015;77(2):S393-S397. 
11. Committee on Trauma, American College of Surgeons. Advanced Trauma Life Support (ATLS®) Student Course Manual. 10 ${ }^{\text {th }}$ ed. Chicago, IL: American College of Surgeons; 2005.

12. Dammers D, El Moumni M, Hoogland II, Veeger N, ter Avest E. Shoud we perform a FAST exam in haemodynamically stable patients presenting after blunt abdominal injury: a retrospective cohort study. Scand J Trauma Resusc Emerg Med. 2017;25(1):1-8.

13. Samuel AE, Chakrapani A, Moideen F. Accuracy of extended focused assessment with sonography in trauma (e-FAST) performed by emergency medicine residents in a level one tertiary center of India. Adv J Emerg Med. 2018;2(2):e15.

14. Cheung KS, Wong HT, Leung LP, Tsang TC, Leung GK. Diagnostic accuracy of focused abdominal sonography for trauma in blunt abdominal trauma patients in a trauma center of Hong Kong. Chin J Traumatol. 2012;15(5):273-8

15. Fornell Pérez R. ¿Eco-FAST o tomografía computarizada multidetectora en el paciente hemodinámicamente inestable tras traumatismo de urgencias? Radiología. 2017:59:531-4.

16. Giraldo-Restrepo JA, Serna-Jiménez TJ. Examen FAST y FAST extendido. Rev Colomb Anestesiol. 2015;43(4):299-306

17. Pérez-Calatayud AA, Anica-Malagón ED, Briones-Garduño JC, Carrillo-Esper R. Protocolos de ultrasonido en estado de choque. Rev Mex Anestesiol. 2017;40(1):S252-S254.
18. Smith J. Focused assessment with sonography in trauma (FAST): should its role be reconsidered? Postgrad Med J. 2010;86:285-91.

19. Tsui CT, Fung HT, Chung KL, Kam CW. Focused abdominal sonography for trauma in the emergency department for blunt abdominal trauma. Int J Emerg Med. 2008;1:183-7.

20. Sue K. The occasional ED ultrasound: focused assessment with sonography for trauma (FAST). Can J Rural Med. 2015;20(1):33-9.

21. Lee BC, Ormsby EL, McGahan JP, Melendres GM, Richards JR. The utility of sonography for the triage of blunt abdominal trauma patients to exploratory laparotomy. Am J Roentgenol. 2007;18:415-21.

22. Tajoddini S, Shams Vahdati S. Ultrasonographic diagnosis of abdominal free fluid: accuracy comparison of emergency physicians and radiologists. Eur J Trauma Emerg Surg. 2013;39:9-13.

23. Instituto Nacional de Estadística, Geografía e Informática [sede web]. México: Instituto Nacional de Estadística, Geografía e Informática. Disponible en: http://www.inegi.org.mx

24. Richards JR, Schleper NH, Woo BD, Bohnen PA, McGahan JP. Sonographic assessment of blunt abdominal trauma: a 4-year prospective study. J Clin Ultrasound. 2002;30(2):59-67.

25. Richards JR, McGahan JP. Focused Assessment with Sonography in Trauma (FAST) in 2017: what radiologist can learn. Radiology. 2017;283(1):30-48 